\title{
Perfil de Velocidade para o Escoamento de Fluido em uma Placa Plana
}

\author{
Velocity Profile fo the Fluid Flow in a Flat Plane \\ Kátia Barros de Lacerda e A. E. A. Amorim \\ Faculdade de Tecnologia de Jahu, CEETEPS \\ CEP 17212-650, Jaú, SP
}

Recebido em 21/02/2001. Aceito em 12/03/2001

\begin{abstract}
Neste trabalho discutimos a abordagem feita para se estudar o conceito de camada limite usando uma distribuição polinomial para o perfil de velocidade no escoamento de um fluido em uma placa plana e comparamos os resultados obtidos na literatura com um outro método onde a distribuição de velocidade é obtida através da série de Taylor em torno da camada limite.
\end{abstract}

In this work we discuss the approach done to study the layer boundary concept using a polinomial distribution for the velocity profile for the flow of a fluid in a flat plate and we compare the results obtained in the literature with another method where the distribution of velocity is obtained trough the series of Taylor around the boundary layer.

\section{Introdução}

Nos livros-textos de Mecânica dos Fluidos utilizados na graduação, um assunto de especial relevância para as Engenharias e a Física, trata sobre o escoamento de fluidos através de corpos submersos.

De uma maneira geral, os livros abordam inicialmente o conceito de camada limite, introduzido por Prandtl[1], em 1904, que sugeriu que a análise do escoamento do fluido, em torno do objeto, pudesse ser feito em duas regiões: uma próxima ao objeto onde os efeitos do atrito são muito importantes, e uma externa, onde o atrito pode ser desprezado. O contorno que delimita estas regiões chama-se camada limite.

Assim, os livros abordam inicialmente o estudo do escoamento do fluido sobre uma placa plana, onde se discute a formação da camada limite. A análise qualitativa deste processo é feita usando o conceito de linha de corrente. Nas linhas adjacentes à placa, aparece uma força de interação entre o fluido e a placa, que produz uma redução na velocidade de escoamento destas linhas. Em virtude da diferença de velocidade entre estas linhas com as linhas de corrente adjacentes do fluido, ocorre o cisalhamento sobre um elemento de fluido tomado nesta região. A forma como ocorre o cisalhamento do fluido está associada com a viscosidade do fluido.

À medida que nos afastamos da placa, o cisalhamento diminui. O resultado final é uma distribuição de velocidade entre as linhas de corrente, também conhe- cida como perfil de velocidade. A forma analítica exata desta distribuição é desconhecida e, por esta razão, são utilizadas algumas expressões aproximadas nos livrostextos, que satisfazem as condições de contorno do problema. É assumido que a velocidade do fluido, junto à placa é nula. Esta é a hipótese do não escorregamento. A outra condição assume que a velocidade do fluido, num ponto distante da placa, é constante e igual a velocidade do fluido sem a placa.

Embora o procedimento adotado pelos livros-textos seja muito utilizado, do ponto de vista didático, o aluno perde a correlação existente entre os coeficientes de seu modelo matemático com as condições de contorno do problema.

Por outro lado podemos aplicar a série de Taylor ao perfil de velocidade em torno da camada limite, utilizando as mesmas condições de contorno, comparando os resultados com as expressões dos livros.

Neste artigo será feita uma discussão sobre estas metodologias para se obter o perfil de velocidade do escoamento do fluido através de uma placa plana.

Antecipando os resultados, observamos que a solução obtida usando a expansão em série de Taylor é única para expansões de primeira e segunda ordem. Nestas ordens de expansão, a expressão é válida para camadas limites muito estreitas. Para expansões de terceira ordem ou superior, o resultado não é único, apresentando infinitas soluções dependentes dos valores dos coeficientes da série de Taylor. O outro método, pelo fato de ser polinomial não apresenta restrição no resul- 
tado.

Por outro lado, observamos que os coeficientes da expansão estão relacionados com as condições de contorno. Este fato permite ao aluno entender a dependência dos coeficientes do perfil de velocidade com as condições de contorno.

Finalmente, notamos que os perfis obtidos são idênticos na forma. Contudo devemos destacar que o método da série de Taylor impõe um regime de validade para a aplicação do perfil de velocidade para camadas limites estreitas enquanto que o método do polinômio não faz qualquer restrição.

O trabalho está organizado na seguinte forma: na seção seguinte discutimos algumas considerações iniciais e apresentamos o modelo. Na última seção apresentamos as conclusões e resultados principais.

\section{Camada limite}

As camadas do fluido, num escoamento real, têm a sua velocidade relativa à placa $\mathrm{v}(\mathrm{y})$ afetada por tensões de cisalhamento provocadas pelas interações existentes as partículas do fluido que percorrem distintas linhas de corrente. De acordo com Prandtl, identificamos uma região na qual o fluido pode ser tratado como ideal, já que a velocidade relativa entre as linhas de corrente é a mesma, e uma outra região na qual a viscosidade desempenha um papel fundamental.

Devido ao atrito existente entre o fluido e a placa, o escoamento da camada de fluido diminui até parar. Esta hipótese do não escorregamento do fluido pressupõe que a velocidade da camada do fluido na placa é nula $v(0)=0$.Evidências experimentais comprovam este fato[2].

À medida que nos afastamos da placa a velocidade do fluido cresce rapidamente até atingir a velocidade do escoamento inicial $v_{o}$, a uma distância $\delta$ da placa, definindo a espessura da camada limite naquele ponto da placa.

Nesta situação a velocidade do fluido é idêntica a velocidade de escoamento do fluido fora da camada limite $v(\delta)=v_{o}$.

Alguns livros assumem que a variação da velocidade, na borda da camada limite é desprezível,

$$
\left.\frac{\partial v}{\partial y}\right|_{y=\delta}=0
$$

utilizando tal equação como condição de contorno. Contudo, devemos frisar que, em um estudo mais realista, as condições de contorno estão restritas ao escoamento do fluido junto à placa $(v(0)=0)$ e fora da camada limite $\left(v(\delta)=v_{o}\right)$.

Tais resultados podem ser resumidos como mostra a Figura 1.

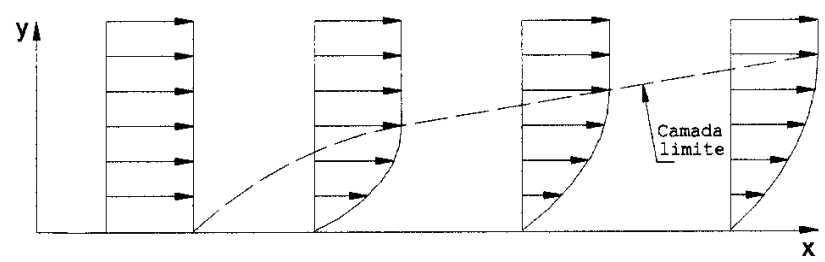

Figura 1. Camada limite e o perfil de velocidade do fluido. As flechas indicam a magnitude da velocidade do fluido. A linha curva e tracejada representa a camada limite. A linha curva e contínua, que delimita as flechas, expressa o perfil de velocidade do fluido.

Contudo, a forma exata do perfil da velocidade é desconhecida, sendo conhecido apenas os seus valores numéricos através da solução de Blasius[3]. Em função disto, alguns autores[3] escrevem o perfil de velocidade como uma função polinomial.

Para um estudo inicial sobre a forma aproximada do perfil de velocidade, os livros-textos assumem uma distribuição das velocidades como uma curva de segundo grau na forma

$$
v(y)=A y^{2}+B y+C,
$$

onde A e B são determinadas pelas condições de contorno já mencionadas.

Com as condições de contorno impostas na Eq.2, temos

$$
\begin{gathered}
v_{o}=A \delta^{2}+B \delta, \\
C=0,
\end{gathered}
$$

e

$$
2 A \delta+B=0 .
$$

Com estes resultados, as constantes se escrevem

$$
A=-\frac{v_{o}}{\delta^{2}}
$$

$$
B=2 \frac{v_{o}}{\delta},
$$

de forma que o perfil de velocidade é dado por

$$
v(y)=v_{o}-\frac{v_{o}}{\delta^{2}}(\delta-y)^{2} .
$$

Observe que nesta dedução o resultado final do perfil expressa a dependência da velocidade em função da coordenada y e, à primeira vista, a Eq.8 não apresenta de forma clara a dependência das condições de contorno no perfil de velocidade.

Para efeito comparativo iremos deduzir o perfil de velocidade usando a série de Taylor. Considere agora uma expansão do perfil de velocidade em torno da camada limite, até a segunda ordem, conservando um ponto fixo na placa, como mostra a Figura 2. 


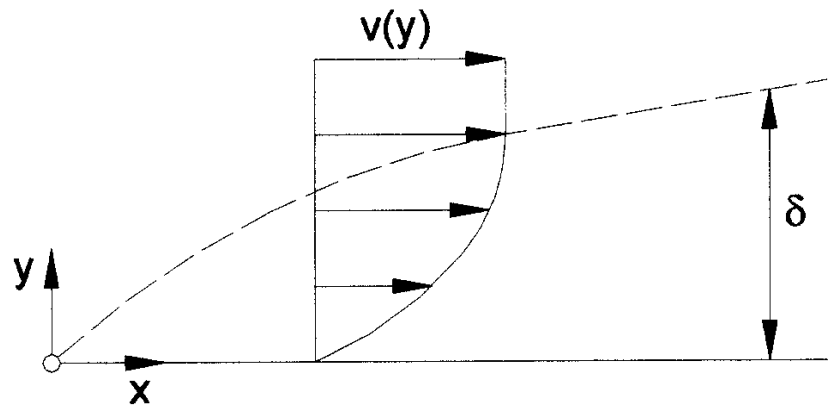

Figura 2. Expansão do perfil de velocidade em torno de um ponto da camada limite.

Assim

$$
v(y)=v_{o}+\left.\frac{d v}{d y}\right|_{y=\delta}(y-\delta)+\left.\frac{1}{2} \frac{d^{2} v}{d y^{2}}\right|_{y=\delta}(y-\delta)^{2}
$$

Aplicando as condições de contorno temos que o segundo termo do lado direito da equação acima é nulo e que, para $y=0$,

$$
v(0)=0=v_{o}+\left.\frac{1}{2} \frac{d^{2} v}{d y^{2}}\right|_{y=\delta} \delta^{2}
$$

Desta forma, temos que o perfil de velocidade é dado por

$$
v(y)=v_{o}-\frac{v_{o}}{\delta^{2}}(y-\delta)^{2},
$$

cujo resultado coincide com a expressão obtida anteriormente na Eq. 8 usando o método do polinômio. Contudo, a validade do resultado dado pela Eq.11 está restrita a camadas limites estreitas, devido a ordem de expansão considerada.

Por outro lado, truncando a série até termos de terceira ordem e aplicando as mesmas condições de contorno,

$$
0=v_{o}+\left.\frac{1}{2} \frac{d^{2} v}{d y^{2}}\right|_{y=\delta} \delta^{2}-\left.\frac{1}{6} \frac{d^{3} v}{d y^{3}}\right|_{y=\delta} \delta^{3},
$$

o perfil de velocidade do fluido se escreve

$$
v(y)=v_{o}+\left.\frac{\delta^{2}}{2} \frac{d^{2} v}{d y^{2}}\right|_{y=\delta}\left(\frac{y}{\delta}-1\right)^{2}+\left(v_{o}+\left.\frac{\delta^{2}}{2} \frac{d^{2} v}{d y^{2}}\right|_{y=\delta}\right)\left(\frac{y}{\delta}-1\right)^{3}
$$

Expandindo as potências e separando as expressões em termos de potência de $y / \delta$ o perfil se escreve

$$
\begin{aligned}
v(y)= & \left(3 v_{o}+\left.\frac{\delta^{2}}{2} \frac{d^{2} v}{d y^{2}}\right|_{y=\delta}\right) \frac{y}{\delta}-\left(3 v_{o}+\left.\delta^{2} \frac{d^{2} v}{d y^{2}}\right|_{y=\delta}\right)\left(\frac{y}{\delta}\right)^{2}+ \\
& \left(v_{o}+\left.\frac{\delta^{2}}{2} \frac{d^{2} v}{d y^{2}}\right|_{y=\delta}\right)\left(\frac{y}{\delta}\right)^{3} .
\end{aligned}
$$

Observe que com séries de terceira ordem ou superiores o perfil de velocidade $v(y)$ é indeterminado, pois depende do valor da segunda derivada da velocidade. Desta forma, este tratamento têm validade para camadas limites estreitas se é utilizada uma solução exata. Para ordens mais elevadas, a distribuição da velocidade fica indeterminada.

Contudo, Shames propõe uma solução particular para este caso de forma que termos da segunda ordem sejam nulos[5]. Portanto, partindo da forma polinomial, o perfil de velocidade obtido é

$$
v(y)=v_{o}\left(\frac{3 y}{2 \delta}-\frac{y^{3}}{2 \delta^{3}}\right) .
$$

Este mesmo resultado é obtido, partindo da Eq.14. Desta forma, pelo fato do coeficiente de segunda ordem ser nulo, então

$$
\left.\frac{d^{2} v}{d y^{2}}\right|_{y=\delta}=-\frac{3 v_{o}}{\delta^{2}}
$$

Substituindo na expressão, o perfil de velocidade reduz a

$$
v(y)=v_{o}\left(\frac{3 y}{2 \delta}-\frac{y^{3}}{2 \delta^{3}}\right)
$$

O resultado é o mesmo que o obtido por Shames, considerando um polinômio. Observe que em ambos os casos estudados, as expressões para a velocidade do fluido são equivalentes, mostrando que os métodos são equivalentes. Contudo a abordagem feita utilizando expansão em séries de Taylor para o perfil de velocidade é mais natural. Os coeficientes apresentam um conteúdo físico e matemático bem claro, ressaltando que o limite de validade do perfil depende da ordem de grandeza considerada. Para ordens elevadas, o perfil 
admite várias soluções e a solução exata é encontrada para camadas limites estreitas.

\section{Conclusões}

Neste trabalho, foi efetuado um estudo sobre a abordagem utilizada nos livros sobre o conceito do perfil de velocidade para um fluido escoando por uma placa plana. A abordagem feita através da expansão do perfil de velocidade em torno da camada limite, utilizando a série de Taylor, torna mais clara ao aluno, permitindo que o mesmo avalie a validade da solução obtida em termos da ordem considerada. Termos de terceira ordem ou superior ficam indeterminadas. Para termos de segunda ordem, a solução é exata. A escolha de funções polinomiais para o perfil de velocidade não permite que se tenha uma idéia clara da relação dos coeficientes em termos das condições de contorno, além do que não impõe uma restrição sobre a validade deste tratamento, induzindo o estudante a crer que é válido para camadas limites de qualquer tamanho.

\section{Agradecimentos}

Os autores agradecem o apoio financeiro da FA-
PESP. Um dos autores (AEAA) agradece ao prof. Marcos Shoiti pela confecção dos desenhos e leitura do artigo.

\section{References}

[1] L. Prandtl, L., Über Flussigkeitsbewengung bei sehr kleiner Reibung, Verhandl. des III Intern. Math.-Kongr., Heidelberg, 1904.

[2] Rui Carlos Camargo Vieira, Atlas de Mecânica dos Fluidos, p. 109, Editora Edgard Blücher Ltda. 1971.

[3] Willian S. Janna, Introduction to Fluid Mechanics, third Ed., International Thomson Publishing, p. 697, 1993.

[4] Ennio Cruz da Costa, Mecânica dos Fluidos, Editora Globo, 1973.

[5] Irving H. Shames, Mecânica dos Fluidos, Editora Edgard Blücher Ltda,Volume I e II, 1973. Tradução Mauro O.C. Amorelli.

[6] Dayr Schiozer, Mecânica dos Fluidos, Editora Argentina, 1990.

[7] Victor L. Streeter e E. Benjamin Wyllie, Mecânica dos Fluidos, Editora McGraw-Hill do Brasil Ltda, 1982. Tradução de Milton Gonçalves Sanches. 